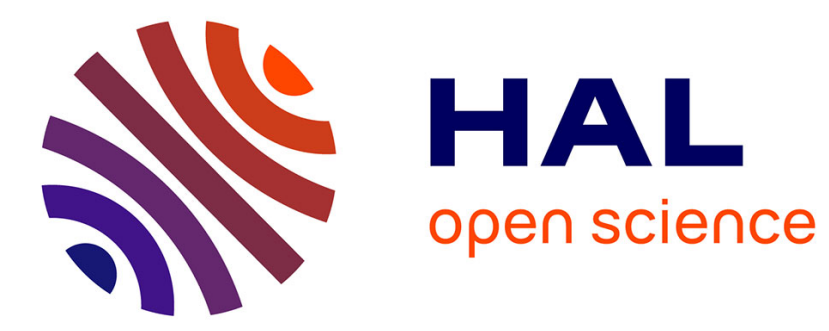

\title{
De la démo-cratie en Amérique
}

Claude Rosental

\section{To cite this version:}

Claude Rosental. De la démo-cratie en Amérique. Actes de la Recherche en Sciences Sociales, 2002, 141-142, pp.110-120. halshs-00007842

\section{HAL Id: halshs-00007842 https://shs.hal.science/halshs-00007842}

Submitted on 17 Jan 2006

HAL is a multi-disciplinary open access archive for the deposit and dissemination of scientific research documents, whether they are published or not. The documents may come from teaching and research institutions in France or abroad, or from public or private research centers.
L'archive ouverte pluridisciplinaire HAL, est destinée au dépôt et à la diffusion de documents scientifiques de niveau recherche, publiés ou non, émanant des établissements d'enseignement et de recherche français ou étrangers, des laboratoires publics ou privés. 


\section{De la démo-cratie en Amérique. Formes actuelles de la démonstration en intelligence artificielle. Claude Rosental}

(C) Copyright Claude Rosental, 2002.

Version préliminaire de l'article suivant ${ }^{1}$ :

C. Rosental, «De la démo-cratie en Amérique. Formes actuelles de la démonstration en intelligence artificielle ", Actes de la recherche en sciences sociales, 141-142, mars 2002, pp. 110-120.

Cet article vise à montrer comment s'est instauré aux Etats-Unis un régime démocratique, fondé sur le recours en sciences à une forme particulière de démonstration, les " démos ». Si l'un de nos objectifs est précisément de décrire les pratiques auxquelles le terme « démo » fait référence, en montrant qu'il s'agit d'un objet de premier plan pour l'analyse sociologique, il est toutefois possible d'en fournir immédiatement un premier aperçu.

« Démo » est une expression couramment employée par les chercheurs en intelligence artificielle et en logique informatique pour désigner un huis clos où un démonstrateur commente le fonctionnement d'un dispositif (par exemple un logiciel informatique ou un robot), destiné à illustrer la valeur et/ou la validité d'un formalisme, d'une méthode, ou d'une approche spécifiques. Il s'agit d'une forme particulière de démonstration, que des chercheurs peuvent utiliser tout autant pour tenter d'obtenir le financement d'un projet de recherche par un industriel que dans le cadre d'échanges savants.

Afin de mettre en lumière les tenants et les aboutissants du recours aux démos, nous exploiterons une fraction des résultats d'un programme de recherche dans lequel nous nous sommes engagé depuis plusieurs années, qui vise le développement d'une sociologie historique des formes de démonstration ${ }^{2}$. Nous partirons plus particulièrement d'une enquête sur les conditions de développement à la NASA, au cours de ces dix dernières années, d'un logiciel "révolutionnaire » dénommé Amphion. Il s’agit d’un programme destiné à la préparation des missions spatiales, servant plus spécifiquement à la définition des trajectoires des satellites de la " conquête de l'espace ». Ce logiciel inédit a été notamment utilisé dans la préparation de la mission Cassini, dernière d'une série d'explorations grandioses des planètes les plus reculées du système solaire. L’organisation de cette mission, décidée en 1989, prévoyait le lancement en 1997 d'un satellite destiné à l'étude de la planète Saturne et de son environnement pendant au moins quatre ans, à partir de juillet 2004.

\footnotetext{
${ }^{1}$ Attention : Ce texte est destiné exclusivement à un usage privé à caractère académique. D'importantes différences peuvent exister entre cette version et celle finalement publiée. Tout autre usage doit faire l'objet d'une demande écrite préalable auprès de l'auteur. Warning: This text is made available for private academic use only. There might be huge differences between this version and the final published one. Always first ask the author for authorization in writing for any other use.

${ }^{2}$ Ce chantier met en effet au jour des phénomènes plus diversifiés et plus lourds d'enjeux que ceux que l'on associerait $a$ priori à une simple contribution à une sociologie du travail intellectuel. Pour d'autres analyses empiriques et des développements théoriques à ce sujet, voir notamment C. Rosental, « Histoire de la logique floue. Une approche sociologique des pratiques de démonstration ", Revue de Synthèse, vol. 4, n 4, octobredécembre 1998, pp. 575-602.
} 
Notre analyse du déroulement de ce projet partira des entretiens, des observations sur le terrain, des correspondances et des archives électroniques que nous avons pu recueillir entre 1993 et 2001. Nous commencerons par présenter les grandes lignes et les conditions de développement du projet pris pour objet. Dans un deuxième temps, nous analyserons plus particulièrement la nature et les enjeux du travail démonstratif déployé par les acteurs. Nous tâcherons alors de mettre en évidence leurs usages des démos, ainsi que les contraintes et les conditions de possibilité propres à cet exercice. Enfin, nous étudierons les dynamiques capitalistes qui caractérisent cette activité, et le type de régime démo-cratique auquel elle aboutit.

Cette démarche va nous permettre de documenter le fonctionnement du champ de la recherche en intelligence artificielle et en logique informatique de la Silicon Valley dans la région de San Francisco, mêlant chercheurs, universitaires, entrepreneurs et représentants de diverses institutions militaires. Nous verrons notamment en quoi les démos constituent l'une des principales sources de mise en forme de leur activité et de leurs relations.

\section{De Thèbes à Saturne}

Dans la mythologie grecque, Amphion, fils de Zeus, avait utilisé sa lyre magique pour amener les pierres se trouvant autour de Thèbes à former les murs de la cité. De manière analogue, le logiciel Amphion avait été développé pour planifier et analyser la trajectoire d'un satellite, en rassemblant des informations et des programmes élémentaires contenus dans une base de données de l'agence spatiale américaine sur le système solaire et sa dynamique, la base «SPICE " ${ }^{3}$. Ce programme informatique pouvait effectuer les calculs utiles à la détermination des positions respectives des corps célestes et d'un engin spatial à un instant donné $^{4}$. Dans ses versions avancées, il permettait aux utilisateurs de visualiser les trajectoires simulées sous la forme d'animations vidéo, et de formuler, à l'aide de diagrammes, les problèmes relatifs à l'optimisation de la position du satellite dans l'espace ${ }^{5}$.

Astronomes et astrophysiciens participant à la définition et à l'exploitation des missions spatiales entraient dans la liste des premiers utilisateurs visés par les concepteurs d'Amphion. Ces scientifiques devaient en effet concilier et traduire en un plan de vol leurs objectifs et leurs contraintes d'observation de divers phénomènes.

Avant le développement d'Amphion, des calculs et des programmes ad hoc à usage unique étaient élaborés pour chaque mission spatiale. Amphion avait ainsi pour objet, entre

\footnotetext{
${ }^{3}$ Voir M. Lowry, A. Philpot, T. Pressburger, I. Underwood, R. Waldinger, M. Stickel, « Amphion: Automatic Programming for the NAIF Toolkit », NASA Science Information Systems Newsletter, Issue 31, Feb. 1994, pp. 22-25.

${ }^{4}$ Voir M. Stickel, R. Waldinger, M. Lowry, T. Pressburger, I. Underwood, « Deductive composition of astronomical software from subroutine libraries ", Proceedings of the 12th International Conference on Automated Deduction (CADE-12), Nancy, France, June 1994, pp. 341-355. M. Lowry, A. Philpot, T. Pressburger, I. Underwood, «Amphion: Automatic Programming for Scientific Subroutine Libraries », Proceedings of the 8th International Symposium on Methodologies for Intelligent Systems, Charlotte, North Carolina, Oct. 16-19, 1994, pp. 326-335. M. Lowry, A. Philpot, T. Pressburger, I. Underwood, " Amphion: Specification-Based Programming for Scientific Subroutine Libraries ", Proceedings of the 3rd International Symposium on Artificial Intelligence, Robotics, and Automation for Space, 1994, Pasadena, California, Oct. 1820, pp. 41-44. T. Pressburger, M. Lowry, "Automating Software Reuse with Amphion », NASA Workshop on Software Reuse, Fairfax, VA, Sept. 24-27, 1996.

${ }^{5}$ Voir S. Roach, M. Lowry, T. Pressburger, "Animating Observation Geometries with Amphion », NASA Science Information Systems Newsletter, Issue 35, March 1995, pp. 35-38.
} 
autres, de limiter l'investissement budgétaire et temporel dans la rédaction de logiciels de calcul.

De façon un peu plus précise, le principe de fonctionnement d'Amphion était le suivant. Le logiciel permettait de traduire une question formulée sous forme graphique en un problème de logique. La question pouvait être par exemple la suivante : où se situe l'ombre de la lune Io sur Jupiter pour l'engin spatial Voyager 2 à un instant t (sachant que les différents corps sont en mouvement, et que la lumière se propage à une vitesse connue) ? Un logiciel de démonstration automatique de théorèmes incorporé dans Amphion, baptisé SNARK, procédait à un traitement logique du problème, qui devait être ensuite traduit sous la forme d'un programme informatique ${ }^{6}$. L'activation de ce dernier devait fournir une réponse à la question initiale en mobilisant des données et des sous-programmes de la base SPICE. Cette dernière était gérée par un groupe d'ingénieurs de la NASA, le groupe NAIF $^{7}$ travaillant au Jet Propulsion Laboratory de l’Université Caltech à Pasadena en Californie.

SNARK constituait la lyre magique d'Amphion aux yeux de leurs concepteurs. Les données et sous-programmes fournis par le groupe NAIF correspondaient, dans le cadre de cette représentation métaphorique, aux pierres situées de façon éparse autour de Thèbes.

Ce schéma fonctionnel correspondait à une réalité institutionnelle dans le développement du projet. Ce dernier avait été lancé par un groupe d'ingénieurs et de chercheurs en intelligence artificielle d'un des grands centres de recherche de la NASA, le NASA Ames research center situé à quelques kilomètres de l'Université de Stanford dans la région de San Francisco. A partir de 1993, ce petit groupe, baptisé ultérieurement $\mathrm{ASE}^{8}$, avait progressivement engagé une collaboration avec une équipe de logiciens du département d'intelligence artificielle d'une autre institution de recherche de la Silicon Valley, répondant au nom de SRI. Cette équipe avait déjà développé une version du logiciel de démonstration automatique de théorèmes SNARK. Son adaptation dans ce qui allait devenir le projet Amphion avait constitué la base d'une collaboration avec le groupe ASE.

Si la coopération entre l'équipe de SRI et celle de NASA Ames était centrale dans cette entreprise en 1994, il n’en allait plus de même au fil des années, des évolutions et des ramifications du projet. Bien d'autres groupes avaient été contactés et plus ou moins fortement impliqués. Il est ici inutile d'apporter de plus amples précisions à ce sujet, car notre analyse portera avant tout sur le travail mené par ces deux équipes sur la période 1993-1994. Ce choix suffira en effet à mettre en évidence la plupart des phénomènes ici pris pour objet.

\section{Objectif Saturne démo.}

Le travail de recherche en logique est souvent perçu essentiellement comme une activité solitaire et un exercice de l'esprit caractérisé par une démarche déductive. La collaboration qui s'était engagée à partir de l'automne 1993 entre le groupe de SRI et celui de la NASA était d'une nature toute autre.

Il s'agissait, à l'issue de tests préliminaires jugés prometteurs par les équipes, de tenter d'adapter le logiciel SNARK aux objectifs et aux problèmes spécifiques des chercheurs de la

\footnotetext{
${ }^{6}$ Voir M. Stickel, R. Waldinger, V. Chaudhri, A Guide to SNARK, Technical Note Unassigned, AI Center, SRI International, May 2000.

${ }^{7}$ Abréviation de « Navigation Ancillary Information Facility ».

${ }^{8}$ Abréviation de « Automated Software Engineering ».
} 
NASA. Cette démarche impliquait notamment des rédactions et réécritures de programmes informatiques, des réunions de travail, et dans la mesure où les deux laboratoires concernés étaient distants de quelques kilomètres, des échanges de courriers électroniques parfois quotidiens. Ces derniers portaient en particulier sur l'état d'avancement des recherches, et véhiculaient des demandes et des communications d'informations les plus diverses.

Dans un premier temps, ce projet avait surtout mobilisé deux chercheurs en logique de SRI, ainsi qu'un informaticien et un chercheur en intelligence artificielle à la NASA. Ce dernier était le principal responsable de cette recherche. Plusieurs chercheurs et ingénieurs avaient ultérieurement participé au projet à des degrés divers.

Cette recherche possédait donc une ample dimension collective. Cependant, elle était également caractérisée par un important travail expérimental et d'observation ${ }^{9}$. Lors des premiers mois de la collaboration, les chercheurs de SRI procédaient à des ajustements de SNARK afin de pouvoir résoudre des problèmes astronomiques formulés usuellement dans le cadre de la préparation des missions spatiales (tels que : où se situe l'ombre d'un corps céleste A sur une planète $\mathrm{B}$ pour le satellite d'observation à un instant $\mathrm{t}$ ?). Les expériences portaient notamment sur les choix de stratégies heuristiques adaptées au domaine " d'application » pour le logiciel démonstrateur de théorèmes, sur les formulations des axiomes à même de produire des résultats "satisfaisants". Les chercheurs étudiaient le comportement de versions successives du logiciel sur des cas précis, notamment ses difficultés à trouver des solutions en fonction des formulations des problèmes, et la nature des solutions trouvées quand SNARK en proposait.

Ce travail était très vite allé de pair avec la recherche de quelques «bons » exemples. Pour mériter ce qualificatif, ces derniers devaient posséder plusieurs propriétés. Il devait s'agir de problèmes rencontrés de façon courante dans la préparation des missions spatiales. Mais ces problèmes devaient également être solvables par le prototype du logiciel, et ce plus rapidement qu'à l'aide des ressources matérielles et humaines employées jusqu'alors. Une liste d'une quinzaine d'exemples « probants » avait ainsi été progressivement dressée.

Autrement dit, le travail des acteurs et la nature même du prototype étaient d'emblée fortement structurés par la perspective de devoir réaliser des démos "convaincantes » à relativement court terme. Ces démos devaient permettre de justifier l'activité de recherche tant au sein des institutions impliquées qu'à l'extérieur - nous y reviendrons. Armés d'une quinzaine d'exemples, les protagonistes étaient a priori à même de montrer en quelques minutes le caractère à la fois rapidement « opérationnel » et « prometteur » de leur recherche.

\section{Une démo constitue-t-elle une expérience publique ?}

Une des premières démos pouvait par exemple se dérouler de la façon suivante. Un ou plusieurs participants au projet exhibaient le fonctionnement de leur prototype en se déplaçant chez ses utilisateurs potentiels, des scientifiques participant à la définition des trajectoires des satellites, ou encore des ingénieurs en informatique de grandes entreprises de haute-

\footnotetext{
${ }^{9}$ Comme l'évoque Jean Largeault, dans J. Largeault, La logique, Paris, PUF, 1993, p. 117 : « Qu'il y ait des logiques détruit l'apriorité de la logique classique, comme la géométrie non euclidienne détruit le caractère d'a priori rationnel de la géométrie euclidienne. [...]. Le pluralisme nous étonne parce que nous avons l'habitude d'associer formel et a priori, et que l'a priori est forcément unique. [...] Cette pluralité déconcerte moins quand on voit dans la logique une science d'observation. Tout dépend de ce vers quoi on décide de diriger l'attention et de ce que l'observation y révèle ».
} 
technologie susceptibles d'être intéressés par une adaptation du logiciel à d'autres fins. Le représentant-démonstrateur (quand il était seul) présentait, depuis un poste informatique, et face à un public de quelques individus, le fonctionnement du logiciel sur la série de cas préalablement sélectionnés.

Pour reprendre un exemple précédemment évoqué, il pouvait ainsi montrer comment calculer la position de l'ombre de la lune Io sur Jupiter pour l'engin spatial Voyager 2 à un instant $\mathrm{t}$ donné. Il présentait comment formuler cette question à l'aide d'un graphique approprié, et soulignait la vitesse de traitement des données par le logiciel pour fournir une réponse sur écran (quelques secondes par exemple), avant de l’imprimer.

A la suite de cette série d'exhibitions, le démonstrateur pouvait inviter les spectateurs à manipuler eux-mêmes le logiciel. Ces derniers s'exerçaient à formuler, en présence du démonstrateur, des problèmes plus ou moins pré-définis selon les cas par le représentantmetteur en scène. Ce dernier pouvait guider l'utilisateur vers des reformulations d'un problème, si le logiciel ne fournissait aucune solution. Ces exercices, comme les exposés préliminaires, étaient souvent marqués par des échanges intenses, en partie déterminés par le scénario et les interrogations mis au point par le démonstrateur.

Qualifier les démos d’expériences publiques, dans la lignée d'un certain nombre de travaux d'histoire sociale des sciences ${ }^{10}$, risquerait de conduire à des malentendus sur la nature des phénomènes en jeu. L'exercice des démos est fort éloigné des tâtonnements d'un travail de laboratoire. Il ne s'agit pas d'expérimenter en présence d'un public le fonctionnement d'un dispositif, mais plutôt de déployer un scénario longuement préparé.

Il s'agit de réaliser un show, ou plus exactement une dé-monstration. Nous employons ce vocable pour désigner un exercice qui se situe à la croisée d'une démarche probatoire (prouver ici qu'un logiciel et une approche de la logique informatique « fonctionnent » bien) et d'une conduite ostentatoire (exhiber un produit et des résultats inattendus, voire inouïs, et en souligner la valeur) ${ }^{11}$. Les démos possèdent en ce sens des propriétés caractéristiques des démonstrations logico-mathématiques plus traditionnelles, réalisés sur support papier ${ }^{12}$.

Comme ces dernières, elles scénarisent la disparition du démonstrateur à certains moments pour insister sur le caractère objectif des énoncés, et parfois sa réapparition à d'autres moments, pour valoriser le démonstrateur en transférant le crédit des résultats à leur auteur. Elles impliquent aussi un long travail préparatoire. Elles possèdent en outre une dimension pleinement scripturale.

Même si les démos ne constituent pas des productions écrites, elles sont en effet fondées sur l'usage d'un script. Si les démonstrations sur support papier doivent conduire le lecteur pas à pas, d'un bout à l'autre d'un texte, les démos guident les regards des spectateurs d'une manière encore plus stricte. Alors que les lecteurs des démonstrations écrites peuvent aisément passer rapidement sur un ou plusieurs pas de démonstration, s’y attarder ou ne pas

\footnotetext{
${ }^{10}$ Ces travaux portent essentiellement sur les sciences expérimentales. Voir notamment D. Gooding, T. Pinch et S. Schaffer (ed.), The Uses of experiment, Cambridge, Cambridge University Press, 1989.

${ }^{11}$ Les enjeux d'une analyse sociologique systématique des pratiques ostentatoires sont considérables. Pour s'en convaincre, voir aussi, dans des domaines très différents de ceux de cette étude, E. Claverie, « Voir apparaître, regarder voir », Raisons Pratiques, vol. 2, 1991, pp. 1-19; L. Daston, « Marvelous Facts and Miraculous Evidence in Early Modern Europe », Critical Inquiry, 18, 1991, pp. 93-124.

12 Voir C. Rosental, «La production de connaissances certifiées en logique: un objet d'investigation sociologique », Cahiers Internationaux de Sociologie, vol. CIX, juillet - décembre 2000, pp. 343-374.
} 
les lire, les démos imposent aux spectateurs un suivi balisé par des exhibitions et des interrogations travaillées, se déployant dans une temporalité incontournable. En ce sens, les démos mettent au jour une dimension fondamentale d'un projet formaliste pour les démonstrations logiques, consistant à tout donner à voir pour permettre à tout un chacun de s'assurer de la légitimité des passages d'un pas à l'autre de la démonstration. Les démos fournissent même des moyens supplémentaires pour réaliser ce projet, de façon plus rigoureuse en un sens que par écrit.

Cependant, les démos conduisent les acteurs de la logique, de façon plus fréquente et plus visible que par le passé, à pratiquer des formes de démonstrations spectaculaires comparables à celles mises en jeu en France et en Angleterre dans les sciences expérimentales au XVIIe siècle, qui servaient de base aux échanges entre le monde savant, les entrepreneurs et les autorités politiques et religieuses ${ }^{13}$. Si l'obtention d'un théorème de portée considérable, au fil d'un long cheminement démonstratif aussi astucieux qu'inattendu, peut être perçu comme spectaculaire pour des logiciens, les démos ont en effet généralement pour objet de mettre en évidence des performances exceptionnelles d'un dispositif et de l'approche à l'origine de son fonctionnement.

\section{Scènes et coulisses des démos}

Compte-tenu de l'ensemble de ses caractéristiques, l'exercice des démos marque fortement la structure de l'espace de travail des chercheurs. Il a en particulier pour effet d'instituer une forme spécifique de partage entre l'espace du laboratoire et "l'extérieur », et de donner une signification particulière à la fermeture de l'univers des centres de recherche. Dans la mesure où la préparation des démos suscite des investissements en temps et en énergie considérables, compte-tenu de l'importance de leurs enjeux pour le maintien de l'activité de recherche, comme nous aurons l'occasion de le préciser, l'espace du laboratoire est finalement transformé en coulisses des démos, alors que "l'extérieur » est constitué en partie d'un ensemble de scènes pour la pratique des dé-monstrations ${ }^{14}$.

Pour réaliser des démos « convaincantes », les participants au projet Amphion étaient par exemple contraints de laisser dans l'ombre ou d'aborder avec beaucoup de précautions certaines incertitudes sur les résultats potentiels de leur recherche. Le travail de laboratoire, marqué par les doutes sur l'issue favorable des expériences, sur leur interprétation et sur le succès des stratégies heuristiques successives, contrastait avec les attitudes assurées, adaptées à la conduite des démos.

De même, les comparaisons entre le temps requis par le logiciel Amphion pour résoudre des problèmes et celui nécessaire à un programmeur pour accomplir les mêmes tâches faisaient l'objet de statistiques amplement travaillées par les équipes de recherche. Le choix des problèmes considérés, la nature des formulations des problèmes sur Amphion, la puissance des ordinateurs utilisés, ou encore l'expérience des programmeurs alimentant les comparaisons constituaient autant de paramètres déterminants pour la production de statistiques favorables au nouveau logiciel ${ }^{15}$. Le simple fait de décider dans le quotidien de la

\footnotetext{
${ }^{13}$ Voir notamment C. Licoppe, La formation de la pratique scientifique, le discours de l'expérience en France et en Angleterre (1630-1820), Paris, La Découverte, 1996.

${ }^{14}$ Autrement dit, les acteurs se retrouvent de fait plongés plutôt dans l'agir dramaturgique que dans l'agir communicationnel. Voir J. Habermas, Théorie de l'agir communicationnel, Paris, Fayard (2 tomes), 1987 (1981) ; E. Goffman, La mise en scène de la vie quotidienne, Paris, Minuit (2 tomes), 1973.

${ }^{15}$ Certains chercheurs en intelligence artificielle sont parfaitement conscients de l'importance de l'opacité des critères de comparaison des performances des dispositifs et du caractère non systématique des approches
} 
recherche de ne pas inclure le temps d'impression des résultats dans les comparaisons temporelles et de ne pas insister sur cette donnée lors des démos constituait un élément important pour renforcer le caractère spectaculaire des dé-monstrations. Ce travail complétait les efforts intenses, fournis jour après jour par les chercheurs de SRI, pour réduire le temps de résolution des problèmes à l'aide de stratégies heuristiques et de formulations axiomatiques astucieuses.

En outre, si pour plusieurs raisons, il pouvait paraître intéressant aux yeux des créateurs de SNARK de présenter Amphion comme une extraordinaire « application » de leur approche de la démonstration automatique de théorèmes, une telle description recouvrait une réalité complexe et difficile à saisir pour qui n'avait pas connu le quotidien du travail de laboratoire. Des adaptations avaient en effet été apportées à SNARK en fonction de la spécificité du domaine de calcul, et notamment du type d'assertions et de questions formulées. Un important travail avait été fourni pour exprimer au mieux les axiomes en jeu. Cette réalité contrastait avec la mise en avant ou les suggestions, en situation dé-monstrative, de l'indépendance du fonctionnement de SNARK par rapport au domaine « d'application ».

L'affirmation du caractère automatique du fonctionnement de SNARK pouvait de même être d'autant plus facilement soutenue lors d'une démo que les modes de formulation de problèmes choisis avaient été testés au préalable dans le quotidien de la vie de laboratoire. La résolution par Amphion de quelques problèmes sélectionnés pouvait être d'autant plus rapide et spectaculaire en situation dé-monstrative que des traductions graphiques infructueuses avaient été réalisées et identifiées comme telles dans le cadre d'un travail préparatoire.

Les démos pouvaient ainsi en grande partie occulter la nécessité de l'acquisition d'un savoir-faire indispensable au bon fonctionnement du logiciel. Par la médiation du travail de formulation graphique de ses questions, l'utilisateur d'Amphion était en fait pleinement acteur de la résolution des problèmes qu'il soumettait. Lorsque le démonstrateur « encadrait » les spectateurs s'essayant à la manipulation du logiciel lors d'une démo, comme ultérieurement lorsque les utilisateurs allaient être "encadrés" par un mode d'emploi du programme informatique, le caractère finalement semi-automatique du fonctionnement d'Amphion pouvait ne pas apparaître clairement. Si l'existence d'une interface graphique dans le logiciel Amphion pouvait être perçu, comparativement au rôle joué par SNARK, comme un élément inessentiel lors d'une démo, on comprend au contraire son importance et les raisons de l'investissement considérable des chercheurs de la NASA dans sa mise au point.

Signalons enfin que le caractère spectaculaire d'une démo du logiciel pouvait tenir également à la dimension apparemment singulière de la démarche des concepteurs. En effet, les démonstrateurs ne se plaçaient pas toujours dans la situation où, afin par exemple de mettre en avant les risques réduits associés à l'investissement dans leur projet, référence était faite à des approches comparables en démonstration automatique de théorèmes, ou dans la réutilisation de logiciels pré-existants. Le fait de ne pas toujours mettre en avant l'important travail de veille réalisé dans leur champ de recherche, notamment par la participation à divers séminaires et conférences et la lecture d'articles pertinents au quotidien, pouvait contribuer à donner l'image d'une approche non seulement singulière, mais plus encore proprement « révolutionnaire ». 


\section{Les usages exploratoires des démos}

L'exercice des démos marquait en fait non seulement la structure de l'espace de travail des chercheurs, mais aussi la gestion de leurs relations. La réalisation d'une démo constituait tout d'abord un mode de présentation de soi. Dans certaines circonstances, un chercheur travaillant dans le champ de l'intelligence artificielle et de la logique informatique pouvait se présenter en formulant d'emblée la proposition suivante: "Bonjour, heureux de vous rencontrer, venez, je vais vous faire une petite démo si vous le souhaitez ».

La première rencontre entre l'équipe de SRI et celle de la NASA s'était opérée sur ce mode. A la suite d'une question posée par courrier électronique par un chercheur du groupe de la NASA concernant l'approche mise en œuvre dans SNARK, les chercheurs de SRI avaient immédiatement proposé un rendez-vous autour d'une " petite démo ». L'engagement d'une collaboration entre les deux équipes s'était jouée en partie sur la réalisation d'une démonstration de ce type. Il en allait de même des partenariats universitaires et industriels qui s'étaient noués autour de leur projet à partir de financements d'origines civiles et militaires.

Les démos constituaient ainsi pour les chercheurs un véritable outil pour rencontrer d'autres acteurs, dans la perspective d'établir des relations d'échanges avec ces derniers. Il s'agissait du reste d'une transaction à part entière : en contrepartie d'un rendez-vous accordé, les démonstrateurs gratifiaient leur hôte d'une exhibition de dispositif. Pour les démonstrateurs, ces formes de dé-monstration représentaient non seulement un aboutissement ou une étape importante dans leurs recherches théoriques et technologiques, mais aussi un moyen de démarchage et de découverte d'institutions susceptibles de leur apporter de nouvelles ressources.

En fait, les démos formaient pour les concepteurs d'Amphion un cheval de Troie dans leur lutte pour assurer le succès de leur projet. Elles pouvaient leur permettre de s'introduire auprès des groupes démarchés puis, dans une certaine mesure, d'en prendre le contrôle. En analysant les réactions suscitées par leurs démos, les démonstrateurs étaient en effet en mesure de recueillir des informations sur les attentes éventuelles des membres de leur public, sur les façons dont ces derniers pouvaient s'approprier le dispositif, sur les ajustements à apporter au prototype pour favoriser son adoption. Cette démarche était essentielle pour transformer les interlocuteurs en futurs utilisateurs, en les faisant au besoin participer par la suite aux développements du projet ${ }^{16}$. L'implication des publics visés dans l'élaboration du dispositif, par l'engagement de diverses formes de partenariats, constituait en fait une ressource précieuse pour adapter tout autant le dispositif aux futurs utilisateurs que son contraire - une pratique ordinairement qualifiée «d'encadrement» ou encore de « formation ». Ultimement, c'est un marché qui était ainsi constitué, le prototype devenant alors un produit bien « ciblé ».

Une dimension essentielle de ce travail de conquête dé-monstrative résidait dans la multiplication des interventions. Les démonstrateurs agissaient en représentants, et même le plus souvent en représentants de commerce, dans la mesure où la «vente » du projet constituait généralement un objectif prioritaire. Ils sillonnaient les routes joignant diverses entreprises, institutions et conférences. Ils démarchaient des entreprises telle Boeing, Apple, ou Microsoft, tentaient d'obtenir des financements d’institutions dépendant du Département

\footnotetext{
${ }^{16}$ Sur ce point, voir aussi S. Woolgar,"Configuring the User : the case of usability trials", in J. Law (ed.), A Sociology of Monsters : essays on power technology and domination, Londres, Routledge, pp. 57-99.
} 
de la défense telles ARPA et exhibaient le fonctionnement de leur dispositif dans différents centres de la NASA ou dans le cadre de séminaires universitaires et de colloques.

On avait ainsi affaire à de véritables campagnes démonstratives. Le travail exploratoire était mené dans une perspective systématique. Il s’agissait de couvrir un certain nombre d'espaces pour créer un effet de masse en matière de circulation de l'information sur le projet. Il n'était en effet pas rare que les décisions d'investissement soient prises à partir de représentations indirectes du fonctionnement d'un prototype. Or les spectateurs des démos constituaient des témoins susceptibles de colporter leurs visions du projet dans des cercles plus étendus que ceux des sites d'exhibition du dispositif.

Dans le cadre de cette activité de démarchage, les démos étaient généralement soutenues par d'autres actions et formes de dé-monstration. Il pouvait s'agir de communications plus traditionnelles, donnant lieu à des présentations hybrides, à mi-chemin entre la démo et l'intervention dans un séminaire. Il pouvait également s'agir de quelques phrases échangées dans des bureaux ou des couloirs avec des managers. Ces phrases étaient réunies en courts argumentaires amplement préparés à l'avance, au point de constituer des répertoires pour les démonstrateurs. Des résumés en une page ou en quelques formules des grandes lignes du projet étaient également distribués, et parfois placés sur des sites web. A côté de ces « clips » de la recherche, destinés à susciter l’intérêt de " décideurs » pressés, des rapports de recherche plus conséquents étaient élaborés et communiqués au sein des organisations.

Il convient de préciser que les campagnes démonstratives n'étaient pas le fait d'individus isolés. Elles faisaient au contraire l'objet d'actions orchestrées et donnaient lieu à la coordination d'un collectif de démonstrateurs. Ces derniers réalisaient généralement leurs démos dans des espaces complémentaires, de façon concertée. Toutefois, il leur arrivait également de se déplacer en nombre pour présenter des aspects complémentaires de leur projet. Cette formule permettait de renforcer le caractère spectaculaire de la démo. Le nombre, tout autant que la qualité et l'expérience des intervenants, pouvaient contribuer à impressionner. Les explorations des institutions procédaient alors de démonstrations de force $^{17}$.

Autrement dit, alors que l'activité démonstrative est généralement perçue comme une activité individuelle, les démos étaient bien loin de constituer des coups démonstratifs isolés. Elles étaient réalisées par vagues pour couvrir un grand nombre d’espaces.

Cette pratique de la dé-monstration n'était pas propre aux participants au projet Amphion. Ces derniers croisaient des semblables dans les institutions démarchées. D’autres démonstrateurs s’y livraient à des exercices analogues.

Dès lors, les représentants-démonstrateurs pratiquaient parfois le trading de transparents pour élaborer leur dé-monstration. Corrélativement, les universités, les instituts de recherche, et diverses organisations civiles et militaires constituaient le théâtre de festivals démonstratifs. Ce phénomène apparemment singulier correspondait en fait à un ensemble de contraintes et de possibilités propres au monde dans lequel évoluait les démonstrateurs. Nous allons maintenant préciser ce point à partir des résultats de l'enquête élargie que le suivi du projet Amphion nous a conduit à mener sur les conditions du travail démonstratif dans le

\footnotetext{
${ }^{17}$ Pour une analyse d'autres usages des démonstrations de force dans un contexte différent, voir C. Mukerji, Territorial Ambitions and the Gardens of Versailles, Cambridge, Cambridge University Press, 1997.
} 
champ de la recherche en intelligence artificielle et en logique informatique dans la région de la Silicon Valley.

\section{Contraintes et conditions de possibilité pour l'exercice des démos}

L'essor de l'informatique depuis les années 1980 n'explique pas à lui seul le développement du recours aux démos, comparativement à d'autres formes de démonstration plus traditionnelles. L'invocation d'un pur déterminisme technique constitue un registre très insuffisant pour rendre compte de ce phénomène.

Tout d'abord, il faut noter que des instituts de recherche comme SRI vivent de contrats industriels civils et militaires, passés souvent en collaboration avec des universitaires ${ }^{18}$. De nombreuses institutions de recherche aux Etats-Unis, et en particulier dans la Silicon Valley, pratiquent également la contractualisation en interne. Les recherches menées à la NASA n’échappent pas à cette réalité. Ainsi par exemple, le responsable du projet Amphion à la NASA travaillait au début des années 1990 pour le compte d'une société de consulting.

Les démos constituent une forme de démonstration particulièrement adaptée à ce contexte. Elles permettent aux chercheurs et aux ingénieurs, lors des évaluations en interne et en externe, d'exhiber des résultats plus " tangibles » que ceux contenus dans des articles très pointus, et ce en quelques minutes. Ces résultats peuvent être appréhendés par des managers pressés et non-spécialistes. Les démos permettent également à ces derniers de suivre les avancées d'un projet, souvent moins perceptibles dans des raffinements théoriques successifs, et ainsi de ne pas perdre confiance dans un programme de recherche se déployant à moyen terme. Elles répondent en outre parfaitement à une activité (et par suite à des évaluations) par projet, plus difficilement saisissable par une analyse périodique d'un volume de publications académiques. Elles complètent aussi les présentations des résultats des recherches sous la forme de rapports volumineux ou sous la forme de résumés, appelant une motivation pour la lecture qui n’est pas toujours présente chez les gestionnaires de la recherche.

L'adoption par des chercheurs de haut niveau d'une casquette de représentant de commerce dans la Silicon Valley tient en d'autres termes à la faible autonomie du champ de la recherche en intelligence artificielle et en logique informatique, et notamment à l'importance au moins aussi grande accordée à l'évaluation de la recherche pratiquée par les sponsors que celle réalisée par les pairs. La prégnance du recours aux démos est étroitement liée au type de relations qui se nouent entre pratiques savantes et démarches entrepreneuriales. Du reste, les identités des acteurs qui évoluent dans ce champ de recherche ne sont pas aussi tranchées que dans d'autres.

Nombre de chercheurs sont à la fois consultants, universitaires, dirigeants ou cadres d'entreprises de haute-technologie et conseillers pour la défense américaine. Tel est le cas notamment de membres d'instituts de recherche possédant un statut de professeur consultant à l'Université de Stanford, ou de professeurs de philosophie de cette même Université qui sont également chefs d'entreprise. Les gestionnaires ont quant à eux souvent une longue carrière derrière eux dans la recherche et l'industrie.

\footnotetext{
${ }^{18}$ Le sigle SRI avait d'ailleurs constitué dans un premier temps une abréviation de Stanford Research Institute, lorsque l'institut gérait les contrats (notamment militaires) de l’Université de Stanford. Après la guerre du Vietnam, l'institut s'est autonomisé, même si les collaborations avec les membres de l’Université de Stanford sont restées très importantes.
} 
Dans ces conditions, on comprend que les démos constituent une forme de communication phare dans ce milieu. Une démo peut être en effet utilisée de façon « universelle » et être capitalisée, dans la mesure où elle est susceptible d'être mobilisée et réemployée dans toutes les facettes de l'activité publique des acteurs : pour présenter des résultats théoriques, un accomplissement technique, ou encore un futur produit de hautetechnologie. Or tel n'est pas le cas par exemple d'un article académique, réservé quant à lui essentiellement aux échanges savants.

Le recours aux démos est à ce point généralisé que le fait de ne pas utiliser cette forme de démonstration devient finalement improbable. Les démos constituent ainsi l'un des opérateurs privilégiés pour la gestion des relations entre les acteurs. Leur existence contribue même au maintien du tissu spécifique liant étroitement recherche, industrie et administration fédérale dans la Silicon Valley, conformément à une réputation acquise depuis plusieurs décennies ${ }^{19}$.

Les démos possèdent il est vrai bien d'autres propriétés que celles que nous venons d'évoquer jusqu'à présent. Elles permettent tout d'abord aux cadres et aux gestionnaires de fonder leur jugement sur les projets non seulement en fonction des avis de tiers, convoqués pour l'occasion en "experts ", mais aussi à partir de réalisations technologiques dont ils peuvent être les propres juges. Le temps limité requis pour assister à une démo spectaculaire d'autant plus spectaculaire que la mise en scène est bien " calibrée » temporellement - fournit aux évaluateurs une occasion unique pour appréhender (ou penser saisir) les projets qui leur sont soumis, indépendamment de l'intervention de garants. Cette donnée est essentielle dans un monde où certains modèles de la science s'avèrent particulièrement irréalistes. Notamment, l'évaluation et la certification des savoirs et des techniques ne s'opèrent pas avec une attention à la limite infinie, mais relèvent au contraire d'une économie très imparfaite en termes de ressources en temps ${ }^{20}$.

Par ailleurs, les démos possèdent des vertus spécifiques face aux représentants des institutions de la défense. Elles s'inscrivent parfaitement dans les traditions des démonstrations spectaculaires et des démonstrations de force qui sous-tendent certaines manifestations militaires. Elles ne se réduisent toutefois pas à des parades. Comme les articles théoriques, elles constituent des ressources pour exhiber des résultats tout en laissant certains aspects des projets dans l'ombre. Elles permettent ainsi de protéger des « secrets » sur les principes du fonctionnement des dispositifs.

Les démos répondent également parfaitement à la logique d’exemplarité qui caractérisent les success stories des consultants, puisqu'elles mettent généralement en scène des cas qui se veulent exemplaires et qui sont érigés en formidables réussites. Cette forme de dé-monstration vient à point nommé dans un monde où la sous-traitance en matière de services (autre terme parfois utilisé pour qualifier l'externalisation ou le recours à la consultance) est généralisée, y compris dans la recherche, et où les acteurs doivent rendre de multiples comptes sur l'objet des contrats.

\footnotetext{
${ }^{19}$ Le rôle joué par les démos n'est bien entendu pas contradictoire avec l'existence d'un ensemble d'autres facteurs qui déterminent l'hybridation du milieu. Tel est le cas par exemple de nombreux appels d'offre qui privilégient le financement de partenariats entre universités, instituts de recherche et entreprises. Le niveau des financements proposés par des grands organismes comme ARPA sont tels qu'ils peuvent effectivement intéresser des industriels, et permettre ce type de collaboration.

${ }^{20}$ Voir notamment C. Rosental, « Les travailleurs de la preuve sur Internet. Transformations et permanences du fonctionnement de la recherche. », Actes de la recherche en sciences sociales, 134, septembre 2000, pp. 37-44.
} 
Les démos constituent ainsi un vecteur idéal pour effectuer le passage entre le monde des laboratoires, marqué par les incertitudes qui jalonnent les processus de recherche, et celui des «décideurs » à la recherche des scénarios les plus certains. Les attitudes pleines d'assurance dont les démonstrateurs doivent généralement faire preuve pour répondre à ces attentes, lorsqu'ils commentent leurs démos, participent largement de ce mouvement ${ }^{21}$. A un niveau agrégé, les démos contribuent finalement en tant que telles à véhiculer des représentations irréalistes de la science. Les doutes qui jalonnent les processus de recherche devant être mis de côté, les faits se durcissent, les affirmations se transforment en énoncés sans histoire, et la marche vers le progrès devient inexorable.

\section{Apprendre à dé-montrer}

Si un tel phénomène peut être observé, c'est d'abord parce que l'art des démos s'apprend. L'assurance des démonstrateurs fait partie des attitudes acquises au cours d'un apprentissage qui s’étend sur de longues années.

Cet apprentissage est en partie autodidacte. Les savoir-faire correspondants ne s'enseignent pas dans le cadre de cours magistraux à l'université. Les démonstrateurs les forgent en partie par eux-mêmes en pratiquant les démos. La formation est ainsi permanente et alimentée par le gain en expérience.

Le mimétisme constitue une autre dimension de cet apprentissage. Une transmission corporatiste des savoir-faire en constitue l'un des vecteurs, même si cette dernière ne se réduit pas elle-même à cet aspect. Si les générations qui ont connu les « débuts » de l'informatique au début de leur carrière n'ont pu compter que sur elles-mêmes, il n'en va pas de même des générations suivantes. Pour ces dernières, l'art de la démo peut être transmis aussi bien sous la forme de conseils que d'incitations hiérarchiques. L'encadrement est réalisé en particulier par les chefs de projet et les gestionnaires de la recherche, qui constituent aussi souvent des chefsdémonstrateurs.

Ces derniers sont généralement en première ligne pour inciter les chercheurs à présenter leur projet sous la forme de success stories. Ce souci est du reste déjà manifeste dans les critères de recrutement des instituts de recherche de la Silicon Valley comme NASA Ames. Les qualités requises pour la conduite des démos font partie des aptitudes exigées à l'embauche.

Les jeunes recrues sont généralement en position de développer leur compétence sous la houlette de chefs démonstrateurs. Souvent bons scientifiques, bons gestionnaires, bons chefs de projet et bons représentants, dotés d'un vaste capital relationnel, ces derniers sont à même de déterminer le caractère plus ou moins adapté des scénarios démonstratifs envisagés en fonction des publics visés, et d'identifier les ajustements les plus fins à apporter selon les cas. Car même si les démos possèdent, comme les démonstrations symboliques, un caractère polysémique, au point qu'une même démo puisse être appréhendée sur des modes très différents d'un spectateur à l'autre, il n'en demeure pas moins que leur adaptation aux

\footnotetext{
${ }^{21}$ Ceci explique d'ailleurs que les démos élaborées aux Etats-Unis ne produisent souvent pas les mêmes effets lorsqu'elles sont réalisées en France : face à des pratiques gestionnaires plus méfiantes voire défaitistes, les attitudes des démonstrateurs peuvent être perçues comme suspectes et outrecuidantes, au point d'être contreproductives. Voir notamment C. Rosental, La trame de l'évidence. Sociologie de la démonstration en logique, Paris, Presses Universitaires de France, 2003.
} 
différents publics constitue une dimension importante du travail des démonstrateurs pour en assurer l’efficacité.

La connaissance approfondie des chefs démonstrateurs des institutions, des identités des interlocuteurs de choix, des conjonctures, des biais diplomatiques, des oppositions claniques, des procédures de préparation du " terrain », ou encore des thèses qui séduisent et des propos qui fâchent les uns et les autres, constituent des ressources précieuses pour assurer le succès des manifestations démonstratives. C'est la raison pour laquelle les chefs démonstrateurs sont en mesure de donner des leçons de démo et d'orchestrer l'effort démonstratif. C'est également l'une des raisons pour lesquelles les répertoires démonstratifs possèdent rarement, même si le registre du spectaculaire suggère l'inverse, une dimension singulière. Bien au contraire, les similarités d'un commentaire de démo à l'autre sont notables et trouvent leur source dans les dynamiques collectives qui président à leur élaboration.

\section{La capitalisation des démos en produits finis}

Si les démos font l'objet d'investissements aussi forts de la part des démonstrateurs, c'est aussi parce que les prototypes construits à cette occasion constituent souvent des produits finis. Il est généralement peu aisé d’opérer une distinction entre outil de démo et produit fini dans la mesure où la célérité du développement de l'informatique contribue à plonger les acteurs dans un système transitoire permanent. Les enjeux des démos sont tels que les démonstrateurs tiennent souvent à bien maîtriser le fonctionnement des logiciels avant de les exhiber en public. Il leur apparaît généralement plus efficace de présenter un dispositif dont ils connaissent bien les défauts qu'un outil amélioré quelques jours plus tôt, dont le fonctionnement risque cependant d'être plus incertain. Compte-tenu du temps requis pour bien connaître le comportement d'un dispositif, il est courant que les outils servant aux campagnes de démo se figent en versions d'un logiciel.

Les campagnes de démo du projet Amphion étaient ainsi réalisées autour de versions volontairement " gelées » du logiciel. Les stabilisations du dispositif en versions successives, dotées de fonctionnalités toujours plus appréciables, permettaient aux démonstrateurs d'accréditer l'idée selon laquelle leurs recherches pouvaient apporter des résultats « concrets » dans des délais "raisonnables ». Bien loin de représenter de simples supports d'expériences publiques, les outils de démos pouvaient dès lors faire l'objet de transactions commerciales, être protégés juridiquement, et être capitalisés en produits.

Diverses logiques, instrumentales, juridiques ou autres, gouvernaient en effet l'élaboration des démos dans ses détails les plus infimes (du moins d'apparence). Si nous avons avant tout abordé les éléments déterminant l'exercice des démos en tant que tel, cet aspect ne doit pas non plus être occulté.

\section{Contraintes et possibilités relatives au contenu des démos}

Dans le cadre du projet Amphion, les démonstrateurs ne pouvaient faire abstraction des risques juridiques liés à des affirmations trop triomphantes. La préparation des répertoires démonstratifs s'opérait ainsi en envisageant une mise en danger potentielle des personnes et des biens. Pour les participants au projet, il s'agissait par exemple de savoir si le calcul des trajectoires d'engins spatiaux par le logiciel pouvait conduire à des pertes de vies humaines ou à des sinistres financiers liés à l'échec de missions spatiales, et si la survenue de telles catastrophes pouvait être imputée aux concepteurs du logiciel. Le rôle important joué par les 
avocats dans les grandes entreprises de haute-technologie et leur intervention fréquente dans la formalisation des partenariats constituaient pour les démonstrateurs une incitation forte à la prudence, tempérant leur souci du spectaculaire.

Le réalisation des démos était également parfois contrainte par les logiques de détention de licences de logiciels. Les premières démos d'Amphion nécessitaient ainsi des transports de matériels ou des connections à distance, afin de pouvoir utiliser un logiciel peu distribué et onéreux requis par Amphion. Face cette contrainte, les démonstrateurs devaient s’astreindre à des contournements et adapter leur logiciel et leur démo.

Le contenu des démos était également fonction de phénomènes conjoncturels. Ainsi par exemple, l'élaboration des premières démos d'Amphion était étroitement liée aux difficultés rencontrées par la NASA au début des années 1990. Suite à une série d'incidents qui avaient causé la perte de vies humaines et des problèmes dans l'accomplissement d'explorations spatiales de grande envergure, les démonstrateurs avaient dû mettre en évidence diverses vertus de leur dispositif, telles l'offre d'une meilleure fiabilité, de meilleures conditions de sécurité, et d'une réduction du coût de préparation des missions. Les scénarios des démos insistaient donc sur l'automatisation de la production de logiciels de calcul, limitant les interventions humaines et " par suite » les risques d'erreurs et les coûts liés au recours à une main d'œuvre qualifiée $e^{22}$.

Le contenu des démos était également déterminé en partie par les logiques de reconnaissance des contributions de chacun ou de concurrence dans la redistribution du crédit. Dans le cas du projet Amphion, les alliances et les rapports de force étaient tels que les présentations relevant d'un réductionnisme logique n'étaient généralement pas de mise. Les chercheurs les plus ingénieurs ne se trouvant pas en position de faiblesse par rapport aux chercheurs les plus théoriciens ${ }^{23}$, Amphion était le plus souvent présenté comme un événement pas moins technologique que théorique. La paternité et la nature du projet n’étaient donc généralement pas uniquement associées, par un réductionnisme aussi fulgurant qu'habituel, aux logiciens du groupe et au pouvoir de la logique mathématique, même si les variations des publics des démos pouvaient parfois conduire les démonstrateurs à infléchir la répartition du crédit associé aux uns et aux autres.

Les commentaires déployés lors et autour des démos ménageaient de même les partenaires potentiels ou effectifs du projet, tels les programmeurs du groupe NAIF. Une grande attention était portée à la qualification et aux modes de mise en avant des progrès associés au développement du logiciel Amphion. Les acteurs étaient en effet parfaitement conscients du fait que, par les phénomènes de répartition et de capitalisation du crédit qu'elles impliquaient à l'égard des noms propres, les démos, comme d'autres « clips » de la recherche (tels des résumés du projet placés sur des sites web), mettaient en jeu le maintien, le renforcement ou encore la destruction des liens et des échanges noués entre les individus ou les équipes.

\footnotetext{
${ }^{22}$ Pour l'étude d'une dynamique comparable, voir D. MacKenzie, Inventing accuracy : a historical sociology of nuclear missile guidance, Cambridge, MIT Press, 1990.

${ }^{23}$ Ce phénomène est apparemment peu usuel dans l'histoire de l'informatique, où les théoriciens semblent généralement dominants dans les couples théoriciens-ingénieurs, avant leur divorce fréquent. Voir P. Lévy, « L’invention de l’ordinateur », dans M. Serres, Eléments d'histoire des sciences, Paris, Larousse-Bordas, 1997, pp. 761-794.
} 


\section{La capitalisation des dé-monstrations en démo-cratie}

Lorsque nous parlons de capitalisation du crédit sur des noms propres, nous faisons en fait référence non seulement à des individus mais aussi à des groupes (comme le groupe ASE de la NASA) et à des institutions (comme la NASA). L'usage des démos échappe en effet en partie aux démonstrateurs.

Comme nous l'avons déjà montré ${ }^{24}$, les démos constituent des outils importants dans les luttes imparfaitement maîtrisées auxquelles se livrent les partisans de diverses théories et approches de la logique informatique et de l'intelligence artificielle. Elles permettent en effet de mettre en avant la valeur d'une posture savante à la mesure de ses réalisations technologiques ${ }^{25}$.

Cependant, les démos peuvent être également utiles à une institution comme la NASA, notamment pour justifier à l'égard du public et des autorités de l'état les dépenses considérables qu'elle engage. Au cours des années 1990, ce phénomène était d'autant plus notable que la NASA devait répondre d'une série d'incidents et d'accidents tragiques, comme nous l'avons évoqué. Le mot d'ordre était donc d'adapter les démos à un public de néophytes.

Quelques années après le début du projet Amphion, les démos ont été ainsi modifiées pour être accessibles au plus grand nombre, en étant placées sur des sites web. Le public de ces adaptations comprend actuellement des amateurs de sciences, des astronomes amateurs, des élèves et des enseignants de science des écoles et des lycées, et tous ceux qui, au gré des navigations sur Internet, sont conduits à consulter les sites de la NASA.

Ces sites rassemblent des images ou des animations présentant les visions passées, présentes et à venir des planètes à partir des satellites d'exploration, ou encore la dynamique des corps célestes. Ces images et ces clips vidéo constituent des simulations réalistes élaborées par de récentes versions du logiciel Amphion, calculées à partir des données de la NASA sur le système solaire. Les premiers scénarios démonstratifs ont été retravaillés. En particulier, les anciens commentaires et instructions orales des démos ont été en partie modifiés et transcrits sous la forme de petits textes d'accompagnement. Des transparents ont également été récupérés pour être placés sur les sites web.

Ce qui constituait dans un premier temps un instrument de démo, avant de devenir un outil de travail pour la préparation des missions spatiales, a donc été à nouveau transformé en support de démo pour le grand public, et comme nous allons le préciser, en instrument de travail dans les écoles et les lycées. Si la frontière entre support de démo et produit fini est aisément franchie, il en va donc de même pour celle qui séparerait outil d'exhibition et instrument de travail $^{26}$.

\footnotetext{
${ }^{24}$ Voir C. Rosental [1998], op. cit.

25 A titre de comparaison, voir comment les tables et les machines à calculer ont constitué des vecteurs du développement de l'analyse mathématique en Angleterre au XIX ${ }^{\mathrm{e}}$ siècle dans A. Warwick, « The laboratory of theory or what's exact about the exact sciences ? ", dans M. N. Wise (ed.), The Values of Precision - Exactitude, Princeton (NJ), Princeton University Press, 1995, pp. 311-351.

${ }^{26}$ A noter que les rubriques d'aide des logiciels les plus courants utilisent du reste fréquemment des démos programmées pour guider leurs utilisateurs.
} 
L'effort démonstratif des chercheurs de la NASA fait l'objet d'une capitalisation particulière dans le monde éducatif ${ }^{27}$. L'agence spatiale américaine a de longue date privilégié ce public pour mener ses campagnes de promotion et trouver des supporters. Or les démos et leur adaptation sur le web se prêtent fort bien aux démarches de vulgarisation scientifique et aux situations pédagogiques. Dans le cas du projet Amphion, les démonstrateurs ont transposé les scénarios de leur démo en initiations à l'astronomie pour les élèves des écoles et des lycées. En se connectant au site web de la NASA, les enseignants disposent ainsi de supports audiovisuels enthousiasmants pour expliquer la dynamique du système solaire aux élèves placés devant des écrans d'ordinateurs. Les démonstrateurs ont développé des scénarios pédagogiques fondés en partie sur l'actualité de la "conquête spatiale », alimentés par des séries de questions réponses, ponctués de saisies de données et d'animations, et appelant à des échanges entre enseignants et enseignés.

Les scientifiques devenus concepteurs de logiciels pédagogiques appellent les enseignants des lycées à leur faire part de leurs réactions sur les scripts pédagogiques par courrier électronique, afin d'en produire des versions améliorées. L'exercice auquel se livrent les démonstrateurs est ainsi de même nature que celui qu'ils ont pratiqué auparavant. Il s'agit pour eux de recueillir des informations sur les pratiques de leurs futurs partenaires, de faire évoluer à la fois leur logiciel et les pratiques pédagogiques, de constituer un tissu relationnel et un marché autour de leur " produit».

Car les bénéfices de ce travail pour l'institution sont en fait multiples. Comme nous l'avons mentionné, l'adaptation des démos permet de promouvoir l'institution. Des résultats tangibles des dépenses sont ainsi affichés. A côté des scénarios démonstratifs, des FAQs ${ }^{28}$ insistent sur les bienfaits et les retombées des explorations spatiales, sur les réductions drastiques des coûts des missions spatiales, et sur l'attention de la NASA à cette dernière question. Ce message est délivré à un public toujours plus nombreux, drainé notamment par l'attrait pour les animations et le suivi de l'actualité de «l'aventure spatiale », comme en témoigne l'encombrement croissant des sites web.

La multiplication des demandes d'affichage rapide des images prises lors des missions, et le nombre élevé de questions portant sur l'utilisation des budgets de la NASA montrent d'ailleurs que le " grand public » est prompt à demander des comptes dès lors que la parole lui est donnée. Une évolution du rapport des scientifiques au public est à l'œuvre. Les chercheurs se retrouvent presque en première ligne pour gérer les représentations publiques de leur activité. L'extension de leur activité démonstrative possède entre autres pour enjeu l'ouverture d'un droit de regard sur la production des laboratoires, et par suite une démocratisation de la science qui ne se réduit pas à l'accès à ses seuls concepts, dans une banale démarche de vulgarisation.

Cependant, la NASA tire d'autres bénéfices des adaptations des démos. A côté des animations, des pages web sont dédiées à la vente de produits dérivés des missions spatiales, comme par exemple des diapositives, des cartes postales, ou encore des maquettes d'engins spatiaux. La source de promotion de l'institution que représentent les démos constitue ainsi également le support d'activités lucratives, selon des pratiques analogues à celles qui caractérisent l'exploitation des productions cinématographies à Hollywood.

\footnotetext{
${ }^{27}$ Voir notamment J. Friedman, S. Lazzeri, T. Pressburger, « Applying NASA technology to education, a case study using Amphion », the 10th International Conference of the Society for Information Technology and Teacher Education (SITE 99), March 1999, San Antonio, Texas, USA.

${ }^{28}$ Abréviation de « Frequently Asked Questions », traduction littérale de « questions posées fréquemment ».
} 
Si la NASA constitue donc une entreprise scientifique capitaliste, c'est en premier lieu parce qu'elle pratique une capitalisation de l'effort démonstratif fourni en son sein. La capitalisation des démonstrations s'opère dans une temporalité plus longue et possède un aspect plus protéiforme qu'il peut paraître de prime abord. Les chercheurs-entrepreneurs ne sont pas les seuls à capitaliser autour de leurs démonstrations, notamment en élaborant des produits, ou encore en accumulant diverses formes de crédit. Une institution comme la NASA en tire également les bénéfices, comme ses dirigeants chargés de rendre compte de son budget.

En définitive, cette recherche montre clairement qu'un régime démo-cratique bien spécifique, comparativement au modèle de la démocratie tocquevilienne, s'est instauré aux Etats-Unis. Celui-ci consacre le pouvoir non pas tant de la foule, par un droit de regard sur l'univers fermé des laboratoires, que celui des démos et de leurs utilisateurs privilégiés, à savoir les capitalistes de la science. 